\title{
Wind Tunnel Tests on Aerodynamic Forces of Road Vehicles Under Unsteady Wind Conditions
}

\author{
M. Sumida ${ }^{1 *}$ and S. Morita ${ }^{2}$ \\ ${ }^{1}$ Department of Mechanical Engineering, Faculty of Engineering, Kindai University, \\ 1 Takaya Umenobe, Higashi-Hiroshima, 739-2116 Japan \\ "Email: sumida@hiro.kindai.ac.jp \\ Phone: +81824347000; Fax: +81824347011 \\ ${ }^{2}$ Graduate School of Systems Engineering, Kindai University, \\ 1 Takaya Umenobe, Higashi-Hiroshima, 739-2116 Japan
}

\begin{abstract}
This paper describes the results obtained from a wind tunnel test on the aerodynamic characteristics of road vehicles subjected to unsteady wind. In order to study the aerodynamic response of vehicles under atmospheric fluctuations, the velocity of the wind has been simulated in a pulsating state, where vehicles at a constant speed are driving in the air with large wind oscillation. On the other hand, we consider three types of vehicles: two types of the Ahmed model, with slant angles of $0^{\circ}$ and $30^{\circ}$, and the basic rectangular-prism model. The effect of wind oscillation on the drag and lift forces acting on the vehicle models under a pulsating wind condition has been extracted by comparing it to the forces under steady wind conditions. The oscillation of the wind exerts a significant effect on the fluid forces, and the drag and lift forces peculiarly change with time, depending on the body shape of the vehicle.
\end{abstract}

Keywords: Wind tunnel test; road vehicle; fluid force; pulsating wind.

\section{INTRODUCTION}

The purpose of this study is to obtain the fundamental characteristics of the fluid forces acting on vehicles subjected to unsteady wind. To this end, we carry out an advanced wind-tunnel test under pulsating wind conditions, which are not yet known. There is no doubt that the aerodynamic performance of vehicles under unsteady wind is very important for the automobile industry to obtain reductions in fuel cost and increase safety and stability. Nevertheless, the evaluation of the aerodynamic characteristics under unsteady flow conditions, assuming a natural environment, is not easy to perform with a real-car wind tunnel because of the cost of the equipment.

Since the Kyoto Protocol, the conservation of the global environment is actively being discussed and, in several countries, people live in a constant sense of impending crisis. At the Paris conference, which included emergent nations, progress has been made as to how to cope with environmental problems. Accordingly, the time has come for each country to consider seriously how to address this matter. Thus, a planned goal to reduce energy consumption is being pursued in full scale. For this reason, in the automotive industry, the restrictions on gas emissions and fuel consumption are being increased every year.

Furthermore, besides improving the safe performance of road vehicles, the requirements for reducing drag forces are now greater [1]. Consequently, in 
manufacturing facilities, practical tests of road vehicles are carried out [2, 3]. Besides, research and development of aerodynamic technologies are now actively pushed forward by computer-aided fluid dynamics (CFD) and wind tunnel tests. Wind tunnels built recently are becoming larger to test full-scale vehicles [1, 2]. Moreover, they have changed in type, from fixed to movable floor wind tunnels. Thus, wind-tunnel tests are being executed under conditions close to real life situations [4]. However, in these windtunnel experiments, the aerodynamic characteristics of vehicles are only examined at a constant wind speed [1-4].

On the other hand, even if the aerodynamic characteristics could be obtained in the above-mentioned way, the airflow speed of an on-road environment is rarely steady. That is, the vehicles ordinarily encounter air with atmospheric fluctuations. Therefore, the specific fuel consumption in a practical on-road test is usually higher than that obtained in a wind tunnel with steady airflow. On some occasions, an atmospheric wind with a highly varying speed may lead to accidents, such as when vehicles turn sideways $[5,6]$. Research is required to cope with these problems $[1,7,8]$. Thus, regarding the characteristics of the aerodynamic forces acting on vehicles, it is desirable to study them not only under the conventional conditions of steady wind but also under unsteady wind $[3,8-10]$.

In response to these requirements, Kindai University started research on car technologies through an industry-academia collaboration and set up an advanced wind tunnel at the Fundamental Technology for Next Generation Research Institute (KURING) [11]. This is briefly described in a recent paper by the author [12]. By using this wind tunnel, we can systematically investigate the unsteady aerodynamic characteristics of road vehicles under variable wind conditions, which change with time. This wind tunnel is able to generate, in a programmable way, time-dependent airflows such as pulsating, gust, and stepwise-varying winds.

Greenblatt [13] described a method for obtaining large-scale variations in airflow speeds in wind tunnels. Moreover, he presented numerous research examples that have used this approach and analysed a particular method based on louvres with multiple variable vanes. With regard to wind tunnels in Japan generating time-dependent airflows, Yagi [14] reported on their methods and equipment.

In the wind tunnel in KURING, the time-dependent airflow is programmable and can be reproduced on countless occasions. It is possible for us to test various flow cases related to unsteady flows. In this paper, we describe a pulsating-airflow test at the beginning of the research on the aerodynamic characteristics of road vehicles subjected to unsteady airflow. We investigate the different influences of wind oscillations on the fluid forces acting on two types of Ahmed-model vehicles [15].

\section{ADVANCED WIND TUNNEL FOR TESTING AERODYNAMIC CHARACTERISTICS OF VEHICLES}

\section{Wind Tunnel}

The dimensions of the advanced wind tunnel in KURING are shown in Figure 1. The wind tunnel is of a closed air circuit and horizontal installation type. It has a nozzle whose exit is a square cross-section with a $600 \mathrm{~mm}$ side. Depending on the experiment, three types of air circuits can be made by setting up and/or combining the air trunks: closed return type; semi-open return type; and open, no return type. Thus, it is possible for us to use this facility for various studies. 
A great advantage of this facility is that it is equipped with an axial flow blower with both a rotor blade pitch-changeable control and rotational speed control. The axial flow blower has 14 blades and is driven by a $200 \mathrm{~V}$ and $45 \mathrm{~kW}$ three-phase circuit electric motor. Furthermore, the pitch angle of the rotor blades and the frequency of the inverter are changeable within the ranges of $0-42^{\circ}$ and $0-60 \mathrm{~Hz}$, respectively. Wind speed, which may change in various patterns, can be programmed and repeatedly generated by 1) properly adjusting the minimum and maximum pitch-angles of the rotor blades; 2) introducing the interval time of the pitching motion. For steady wind, the available speed ranges from 1.5 to $45 \mathrm{~m} / \mathrm{s}$. Manual operation is also possible.

The contraction section is $1.485 \mathrm{~m}$ in length, and a rate of contraction of approximately $5: 1$. Just behind the exit of the wind-tunnel nozzle, the ground plane was set up to put a vehicle model. The ground plane can be adjusted up and down to scoop the fluid with lower speed near the wall at the nozzle exit.
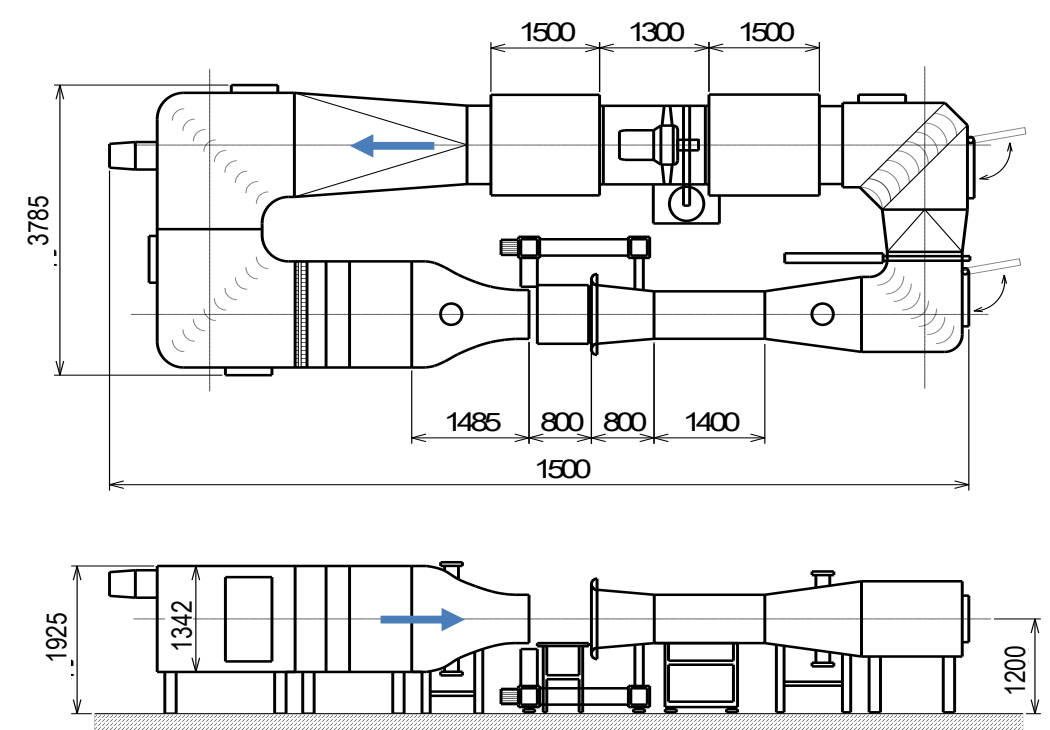

Figure 1. Schematic diagram of a wind tunnel.

\section{Airflow Speed Control System}

The outline of the configuration of the automatic control system to operate the wind tunnel is shown in Figure 2. The system consists of a flow velocity sensor (part I), controls to drive the electric motor and axial flow blower (part II), and a boundary-layer control (part III). Furthermore, the sensors used to measure the several quantities are summarised in Table 1, together with their manufacturer, ranges and accuracies.

The velocity of the airflow issued from the wind tunnel nozzle is measured with both a Pitot tube and a hot-wire anemometer. The Pitot tube is of a standard type, in which the dynamic pressure is measured with a differential pressure transducer (Yokogawa Electric Co., EJA110). Flow velocity is calculated using the wind-tunnel static pressure (Yokogawa Electric Co., EJA310). On the other hand, the hot-wire anemometer is made by KANOMAX JAPAN Inc. (Model 6141). The flow-velocity values obtained from the hot-wire anemometer and the Pitot tube can be properly displayed on the personal computer (PC) monitor screen by switching their outputs. 
(I)

(II)

(III)

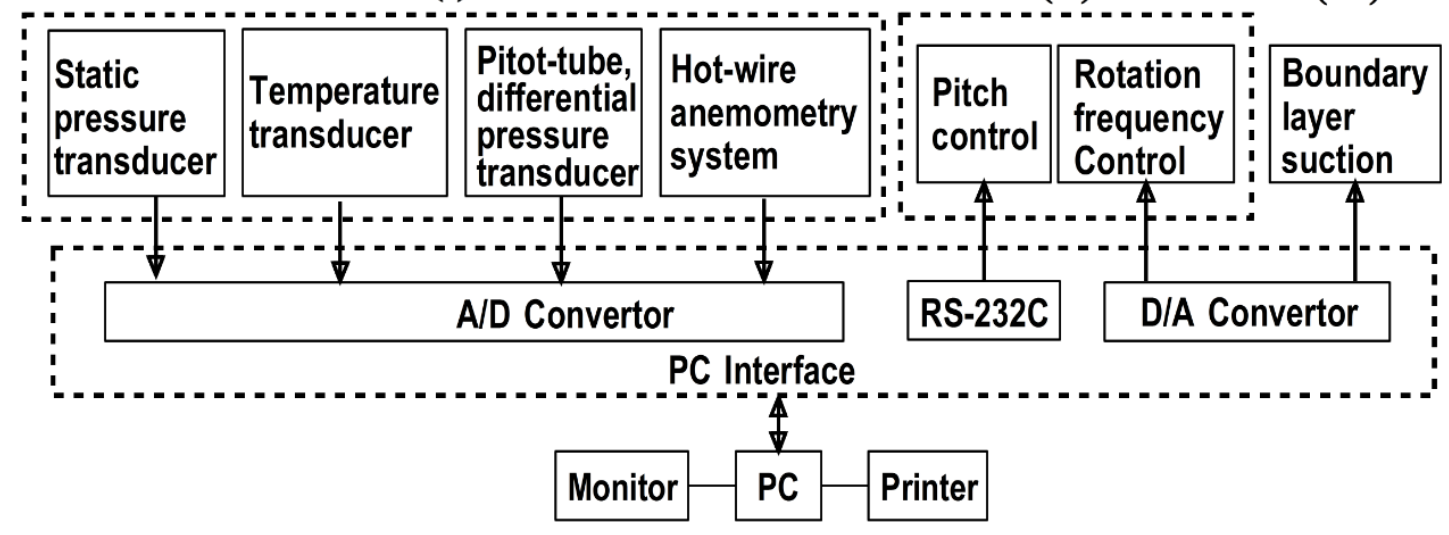

Figure 2. The operating system of a wind tunnel.

Table 1. Measurement sensors with their manufacturer, ranges and accuracies.

\begin{tabular}{|c|c|c|c|}
\hline Quantity & Sensor and type & $\begin{array}{c}\text { Range } \\
\text { (Accuracy) }\end{array}$ & $\begin{array}{l}\text { Manufacturer name } \\
\text { (Product name) }\end{array}$ \\
\hline \multirow[t]{3}{*}{ Airflow velocity } & Pitot tube (JIS standard & $0-50 \mathrm{~m} / \mathrm{s}$ & TSUKUBA RIKA SEIKI, \\
\hline & & $( \pm 0.1 \%)$ & Co. $(\phi 6-\mathrm{LTR})$ \\
\hline & Dynamic pressure & $\begin{array}{c}0-10 \mathrm{kPa} \\
( \pm 0.1 \% \mathrm{FS})\end{array}$ & $\begin{array}{c}\text { Yokogawa Electric Co. } \\
\text { (EJA310) }\end{array}$ \\
\hline Airflow velocity & Hot-wire anemometer & $\begin{array}{l}0-50 \mathrm{~m} / \mathrm{s} \\
( \pm 2 \% \mathrm{FS})\end{array}$ & $\begin{array}{l}\text { KANOMAX JAPAN Inc. } \\
\text { (Model 6141) }\end{array}$ \\
\hline $\begin{array}{l}\text { Velocity } \\
\text { distribution }\end{array}$ & Hot-wire anemometer & $\begin{array}{l}1-50 \mathrm{~m} / \mathrm{s} \\
( \pm 0.2 \% \mathrm{FS})\end{array}$ & $\begin{array}{c}\text { KANOMAX JAPAN Inc. } \\
\text { (System 7000) }\end{array}$ \\
\hline $\begin{array}{l}\text { Aerodynamic } \\
\text { forces }\end{array}$ & Multi-component load cell & $\begin{array}{c} \pm 20 \mathrm{~N} \\
( \pm 0.2 \% \mathrm{FS})\end{array}$ & $\begin{array}{l}\text { Nissho-Electric-Works Co. } \\
\text { (LMC 3502) }\end{array}$ \\
\hline \multirow[t]{2}{*}{ Static pressure } & $\begin{array}{l}\text { Diffusive-type } \\
\text { semiconductor }\end{array}$ & $\pm 10 \mathrm{kPa}$ & JTEKT Co. \\
\hline & $\begin{array}{l}\text { Differential pressure } \\
\text { transducer }\end{array}$ & $( \pm 0.03 \% \mathrm{FS})$ & (DD101K) \\
\hline Static pressure & Digital pressure gauge & $\begin{array}{c}0-2 \mathrm{kPa} \\
( \pm 0.2 \% \mathrm{FS})\end{array}$ & $\begin{array}{l}\text { Okano Works Ltd. } \\
\text { (DMC) }\end{array}$ \\
\hline Temperature & Platinum thermometer & $\begin{array}{c}-50-+150^{\circ} \mathrm{C} \\
\left( \pm 0.2^{\circ} \mathrm{C}\right)\end{array}$ & $\begin{array}{l}\text { OMRON Co. } \\
\quad(\text { E52) }\end{array}$ \\
\hline
\end{tabular}

\section{Mode of Wind Velocity}

In this wind tunnel, we can drive various modes of wind speed to examine the unsteady aerodynamic characteristics of road vehicles subjected to fluctuating wind. Four main types of wind fluctuation are possible: steady, pulsating, gust, and stepwise-varying wind modes. We will explain the first two modes that were executed in this study, as shown in Figure 3.

First, for the conventional steady-wind mode in Figure 3(a), the airflow velocity $V$ of $1.5-45 \mathrm{~m} / \mathrm{s}$ is generated by controlling the rotational speed of the axial flow blower. Here, the rotational frequency is set with the computer and/or is adjusted by manual operation of the control panel. Next, for the pulsating-wind mode in Figure 3(b), there are 
different procedures for rotor blade control and rotational speed control. For the former, the pitch angle of the rotor blade is controlled according to the instructions received from the computer under a constant rotational frequency of the axial flow blower. For the latter, on the contrary, the rotational frequency of the axial flow blower is changed under a fixed pitch angle.

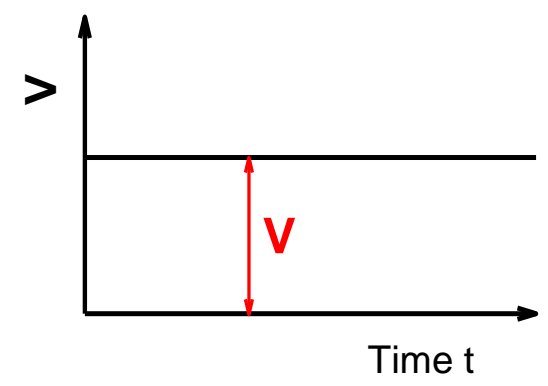

(a) Steady wind mode.

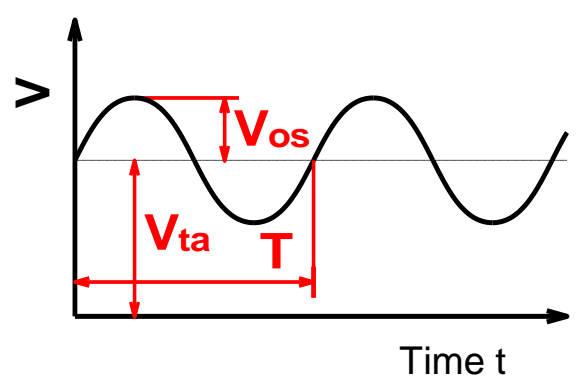

(b) Pulsating wind mode.

Figure 3. Wind modes.

\section{EXPERIMENTAL APPARATUS AND METHOD OF MEASURING FLUID FORCES}

\section{Experimental Apparatus and Measurement Procedures}

Figure 4 shows the schematic of the experiment carried out with the semi-open-type wind tunnel. The drag, side, and lift forces ( $D, S$, and $L$, respectively) applied to the vehicle model are detected by the multi-component load cell (Nissho-Electric-Works Co., LMC 3502). The output voltages of the load cell are amplified with direct current amplifiers (Nissho-Electric-Works Co., DSA 100A). They are converted to digital quantities through a data collection system (Keyence Co., NR-500) and are then processed in the personal computer. The static pressures on the vehicle model are measured using diffusive-type semiconductor differential pressure transducers (JTEKT Co., DD101K) together with digital pressure gauges (Okano Works Ltd., DMC). Additionally, their output voltages are processed with the above-mentioned data collection system.

\section{Vehicle Model}

Figure 5 shows the vehicle models employed in the present experiments. We use the Ahmed-type model [15] with a slant angle of $30^{\circ}$ because, until now, most data [16-22] had been accumulated under steady airflow conditions. The size of the model is such that the blockage ratio of the wind tunnel does not exceed 1/20. Its dimensions are 417.6, 155.8 , and $115.2 \mathrm{~mm}$ in length, width, and height, respectively. Thus, the test model is approximately $1 / 12.5$ of a practical vehicle and $1 / 2.5$ of the Ahmed model [15]. The model is supported by four columns $6 \mathrm{~mm}$ in diameter, as shown in Figure 4.

In the work of Ahmed and Ramm [15], the influence of the rear slant angles on the drag coefficient had been examined. Nevertheless, the model with a slant angle of $30^{\circ}$ was preferred in our study because the drag coefficient is assumed to take a maximum value [15]. It is called model A, as presented in Figure 5(a). Moreover, the model with the slant angle of $0^{\circ}$ and the most basic rectangular prism [23] were employed as objects for comparison. They are named models B and C, respectively, in Figures 5(b)-(c). 


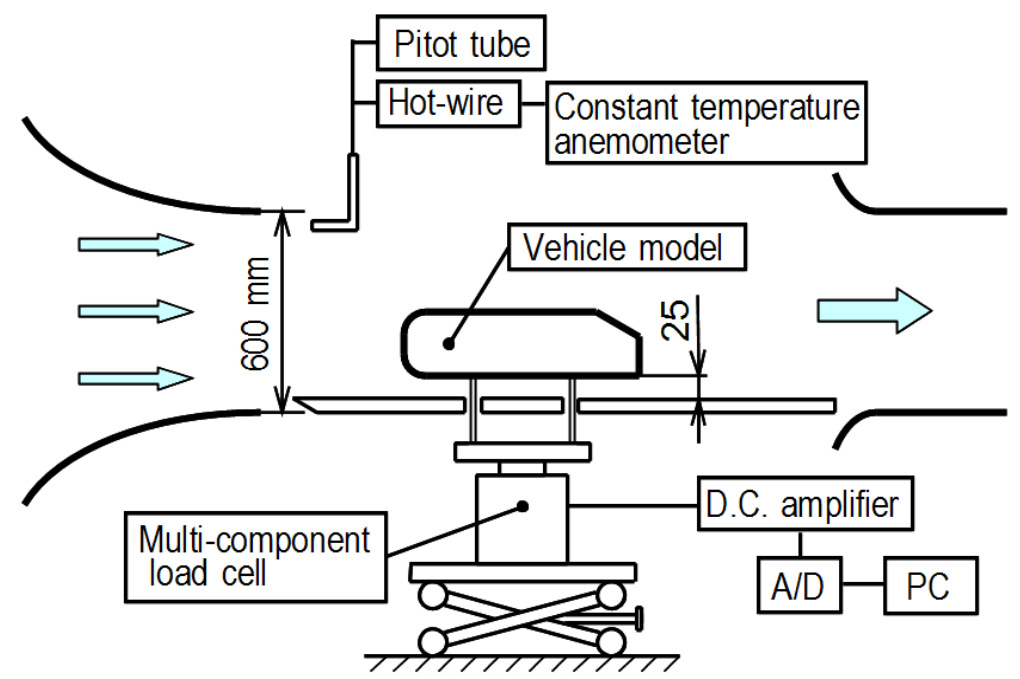

Figure 4. Schematic diagram of experimental apparatus.

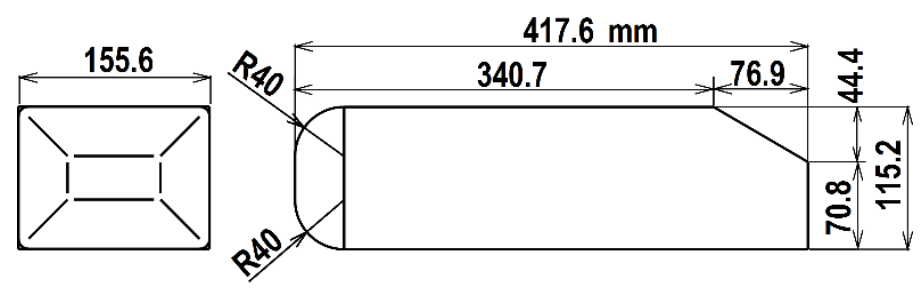

(a) Model A (Ahmed-type model with a slant angle of $30^{\circ}$ ).
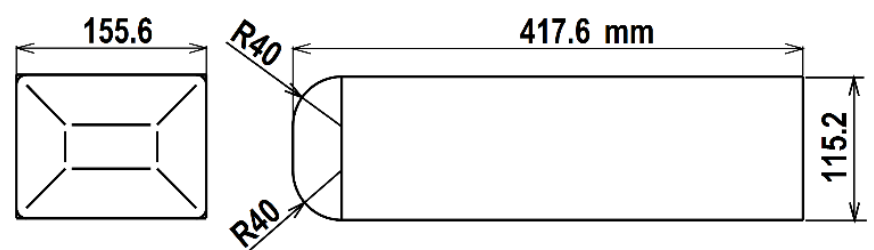

(b) Model B (Ahmed-type model with a slant angle of $0^{\circ}$ ).
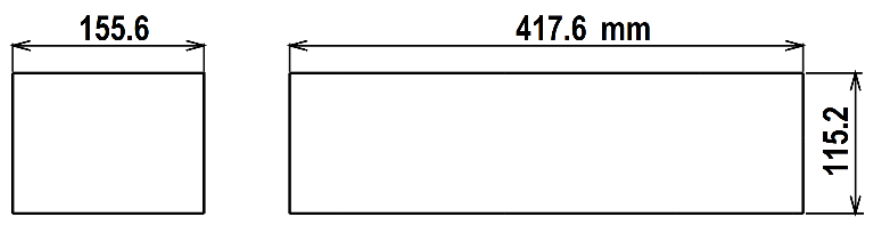

(c) Model C (basic rectangular-prism model).

Figure 5. Road vehicle models. 


\section{RESULTS AND DISCUSSION}

\section{Steady Wind Case}

\section{Velocity distribution in the cross-section of the nozzle exit}

The velocity distribution in the central part of the cross section of the nozzle exit is shown in Figure 6. In the figure, the origin of the coordinate system is the centre of the cross section of the nozzle exit. The $Y$ and $Z$-axes are in the horizontal and vertical directions of the exit plane, respectively. The distribution of the airflow velocity $V$ is provided in a non-dimensionalised form, based on a representative velocity of $V_{a}$. Here, the velocity measurements were performed under a centre velocity of $20 \mathrm{~m} / \mathrm{s}$, where the representative velocity $V_{a}$ is the value averaged in the region with $-200 \leq Y \leq 200 \mathrm{~mm}$ and $-200 \leq Z \leq$ $200 \mathrm{~mm}$. In the nozzle-exit area outside the region illustrated in Fig. 6, measurement of the velocity was executed only on the $X$ and $Y$-axes. In most of the nozzle-exit cross sections, $400 \times 400 \mathrm{~mm}$, the velocity distribution has uniformity within $1 \%$ of deflection. Nevertheless, at the measuring points near the corners, the speed tends to become slightly larger. The turbulence intensity at the centre of the exit plane, at a speed of $20 \mathrm{~m} / \mathrm{s}$, is $0.7 \%$ or less and therefore is sufficiently small.
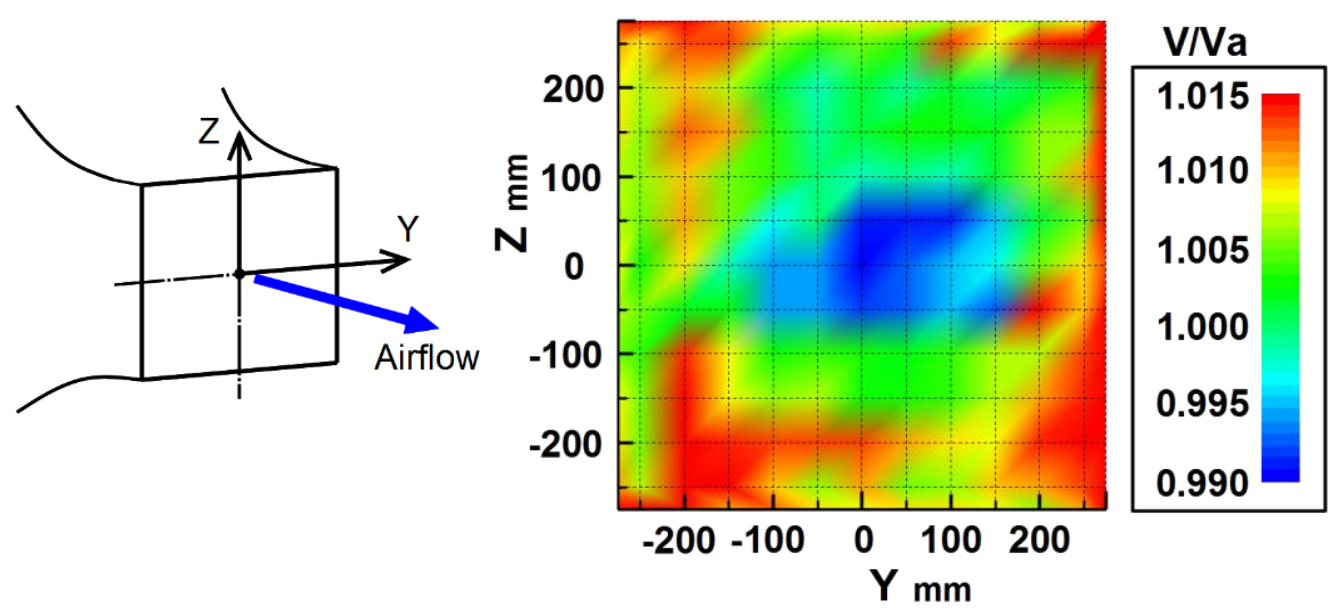

Figure 6. Velocity distribution in the central part of the cross section at the nozzle exit. (with representative velocity, $V_{a}=20 \mathrm{~m} / \mathrm{s}$ ).

\section{Fluid forces acting on road vehicles}

When vehicles run on roads, they experience a fluid force that can be decomposed into three components: drag, side, and lift forces, $D, S$, and $L$, respectively. That is, the drag force $D$ is in the flow direction, and the lift and side forces, $L$ and $S$, are in the direction of the $Z$ and $Y$-axes, respectively. These components are expressed by the following equations and are evaluated using the corresponding non-dimensional coefficients of $C_{D}$, $C_{S}$, and $C_{L}$.

$$
\left(\begin{array}{l}
D \\
L \\
S
\end{array}\right)=\frac{1}{2} \rho V^{2} A\left(\begin{array}{l}
C_{D} \\
C_{L} \\
C_{S}
\end{array}\right)
$$


Where, $\rho$ is the air density, and $A$ is the projected frontal area of the vehicle model. Furthermore, $V$ is the freestream velocity measured at the point shown in Figure 4. Among these coefficients, the drag-force coefficient $C_{D}$ is the most important because it is related to fuel cost and acceleration performance. On the other hand, the side-force coefficient $C_{S}$ influences the crosswind stability, while the lift-force coefficient $C_{L}$ is related to straight-drive and crosswind stabilities.

In this study, we examine the problem that the vehicle is going straight. Therefore, we focus on the drag and lift forces, which exert the greatest influence on the aerodynamic characteristics of vehicles. The results of the drag and lift coefficients for each of the models, which are set up at a height from the ground of $h=25 \mathrm{~mm}$, are shown in Figure 7. The drag coefficient $C_{D}$ of model $\mathrm{C}$, with the four sharp corners in the front, is approximately twice as large as that of models $\mathrm{A}$ and $\mathrm{B}$, with rounded corners, as shown in Figure 7(a). Models $\mathrm{A}$ and $\mathrm{B}$ have the same shape in the front, and $C_{D}$ of model $\mathrm{A}$, with the slant angle of $30^{\circ}$, is approximately $30 \%$ larger than that of model B [15].

On the other hand, the lift coefficients of model B and C, with symmetric upper and lower halves, take a negative value, as depicted in Figure 7(b). This means that the vehicles receive the force in the direction of ground, and the downforce in model $\mathrm{C}$ is approximately $40 \%$ larger than in model B. In contrast, the lift coefficient in model A, with an asymmetric body-shape, has a positive value indicating that the vehicle receives an upward force. For vehicles with a slant angle of $30^{\circ}$, like model A, there occurs a reduction in pressure in the upper part of the rear of the bodies due to a large-scale vortex. Consequently, $C_{D}$ and $C_{L}$ are simultaneously increased, which is well known from the work of Ahmed and Ramm [15]. For models B and C, the flow speed under the body becomes larger. Therefore, it is considered that the negative pressure on the floor side is intensified and $C_{L}$ takes a negative value. In particular, the pressure for model $\mathrm{C}$ decreases further because the flow near the corner of the front body is strongly separated. As a result, it is conceivable that the negative value of $C_{L}$ in model $\mathrm{C}$ increases in magnitude compared to model $\mathrm{B}$. When the wind velocity exceeds approximately $10 \mathrm{~m} / \mathrm{s}$, at a modellength based Reynolds number of $R e=3.15 \times 10^{5}$, the changes in $C_{D}$ and $C_{L}$ for each model become small in relation to the increments in velocity.

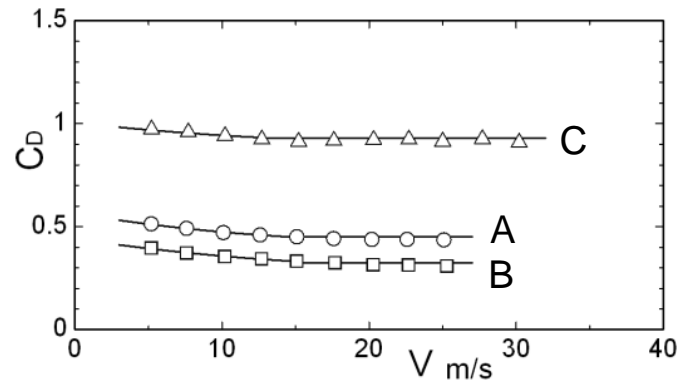

(a) Drag coefficient.

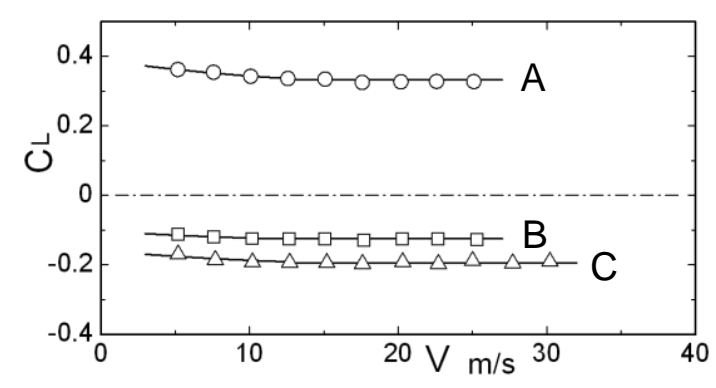

(b) Lift coefficient.

Figure 7. Drag and lift coefficients under steady wind (with A: Model A, B: Model B, C: Model C).

\section{Pressure distribution on the vehicle body}

The results of the pressure distribution on the body surface for model A at $V=20 \mathrm{~m} / \mathrm{s}$ are shown in Figure 8 as a representative example. In the figure, the horizontal axis shows 
the length measured from the front of the model, while the vertical axis indicates the pressure coefficient $C_{p}$ defined by the following expression:

$C_{p}=\left(P-P_{0}\right) /\left(\rho V^{2} / 2\right)$,

Where $P$ is the static pressure on the body surface and $P_{0}$ is the static pressure in the wind tunnel. The pressures on the body surface take a negative value, excluding the central part of the front of the body. The negative values for pressure are larger on the upper and lower sides of the front part and the slant surface of the body. Thus, such a distribution for model A results in a positive value for $C_{L}$. Furthermore, it can be seen from Figure 8 that the pressures on the floor side at $x / L=0.15-0.5$ are a little lower than on the upper side.

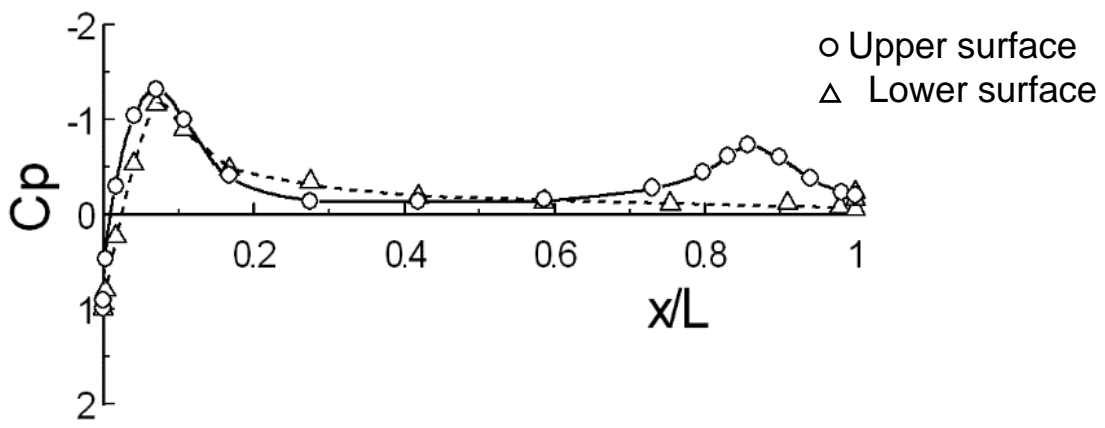

Figure 8. Pressure distribution around the body of model A under steady wind at $V=20$ $\mathrm{m} / \mathrm{s}$.

\section{Pulsating Wind Case}

In this section, we consider the case when road vehicles receive pulsating wind. Then, we discuss the influences of the oscillating wind on the fluid forces due to the vehicle shape. Figure 9(a) to (c) show examples of the results obtained for each model. The experiments were conducted under pulsating wind conditions, i.e., the wind changes with a pulsation period of $T=1.5 \mathrm{~s}$. The vertical axes on the left and right show the drag and lift forces, $D$ and $L$, and the wind velocity $V$, respectively. Here, velocity $V$ is non-dimensionalised by the time-averaged value, $V_{t a}$, of $V$. The wind velocity changes almost sinusoidally with time $t$ and can be simply expressed by the following Eq. (3).

$V(t) \approx V_{t a}+V_{o s} \bullet \sin \Theta$,

Where $\Theta(=\omega t)$ is the phase angle and $\omega$ is the pulsation frequency. The subscripts $t a$ and $o s$ indicate time-mean and amplitude values, respectively. According to this expression, the time-mean and oscillating components of the wind in Figures 9 are $V_{t a}=13.2 \mathrm{~m} / \mathrm{s}$ and $V_{o s}=3.1 \mathrm{~m} / \mathrm{s}$, respectively. They give an amplitude ratio of $\eta=0.235$. The dashed and dotted lines in the figures show the time-mean values.

The time-mean drag force $D_{t a}$ in each model is approximately $10 \%$ larger than the drag force under the steady wind, with the same value as the time-averaged velocity of the pulsating wind. Moreover, the oscillating component $D_{o s}$ is equivalent to approximately $50 \%$ of the time-mean value $D_{t a}$, though the oscillating ratio $\eta$ is approximately 0.24 . Therefore, it is understood that the fluctuation of wind speed has a 
striking effect on the vehicles. Furthermore, the drag force varies with a phase lead from the oscillation of the wind, and the more the shape of the model takes a drag force, the larger the phase difference.

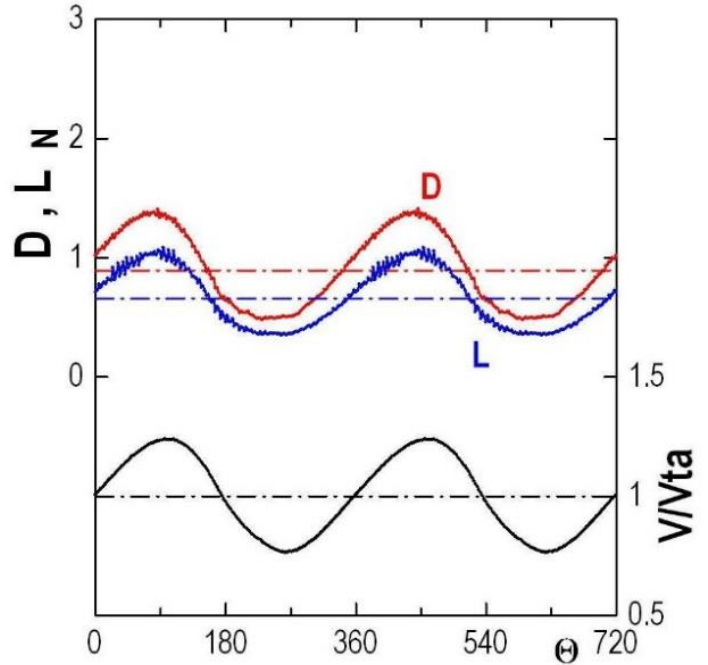

(a)

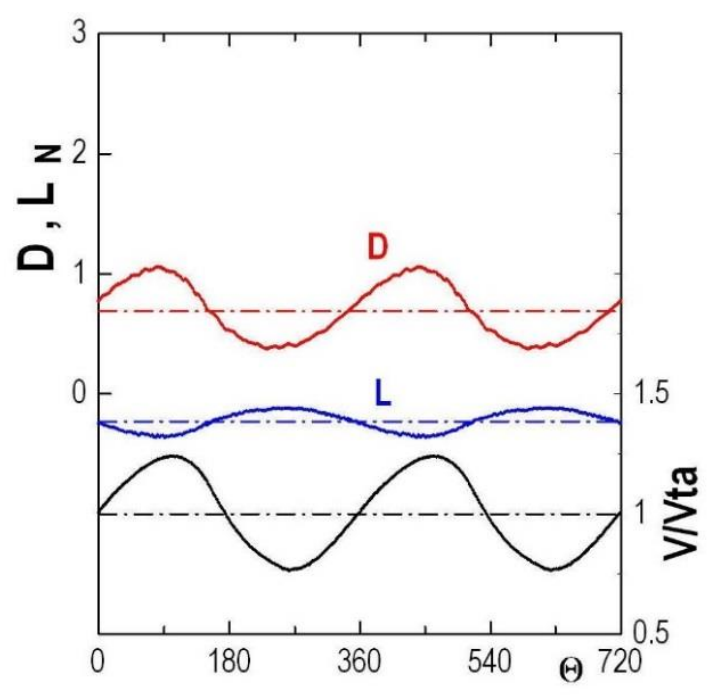

(b)

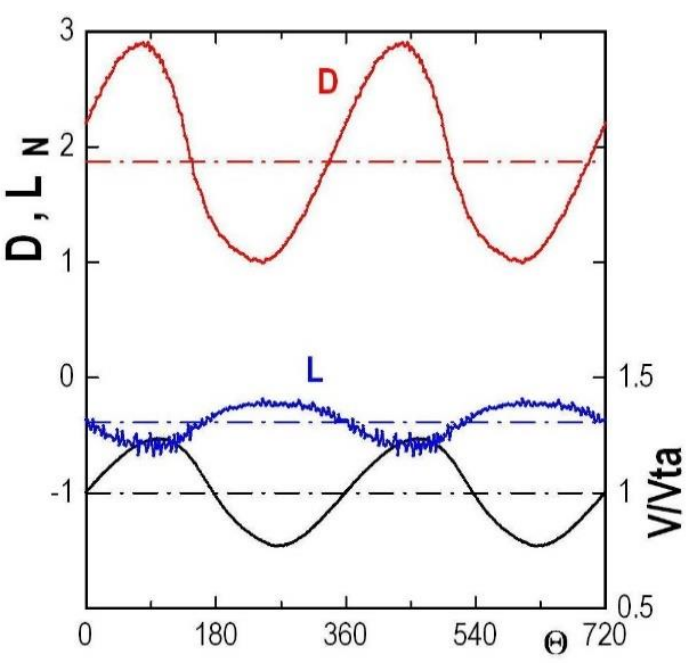

(c)

Figure 9. Changes in drag and lift forces, $D$ and $L$, with time under pulsating wind for (a) model A, (b) model B and; (c) model C. Dashed and dotted lines show the timeaveraged values.

The lift force $L$ of each model under pulsating wind has the same sign as under steady wind. To state it more precisely, model A shows a positive value, while models B and $\mathrm{C}$ show negative values. However, they show a specific periodic change in the lift force against the oscillation of the wind velocity. That is, for models B and C, with symmetric upper and lower halves, the lift force changes in the opposite phase of the oscillating wind. Moreover, $L$ indicates a large absolute value when the wind velocity is high. The amplitude value $L_{o s}$ becomes larger in the following order of models: B, C, and A. The reason could be as follows. In model A, the lift force is increased in the upward 
direction as the vortex motion near the rear body is intensified when the wind speed is greater.

Conversely, for model $\mathrm{C}$, the downward force increases owing to the flow separation that occurs near the lower corner of the front of the body; thus, the amplitude value of the lift force becomes even stronger. The amplitude ratio, $L_{o s} / L_{t a}$, of the lift force is the largest in the case of model C. Under pulsating wind, vehicles with a horn-shaped front body seem to increase oscillations in the pitching moment and to worsen their straight-drive stability.

The relations between the fluid forces and the wind velocity for a cycle are shown in the form of a Lissajous diagram in Figure 10. In the figures, the drag and lift forces, $D$ and $L$, are normalised by the dynamic pressure, $\rho V_{t a}{ }^{2} / 2$, with the time-mean velocity, $V_{t a}$, of the wind. They are expressed in the same manner as Eq. (1), i.e.,

$$
\left.\begin{array}{l}
C_{D}(t)=D /\left(\rho V_{t a}^{2} A / 2\right) \\
C_{L}(t)=L /\left(\rho V_{t a}^{2} A / 2\right)
\end{array}\right\}
$$

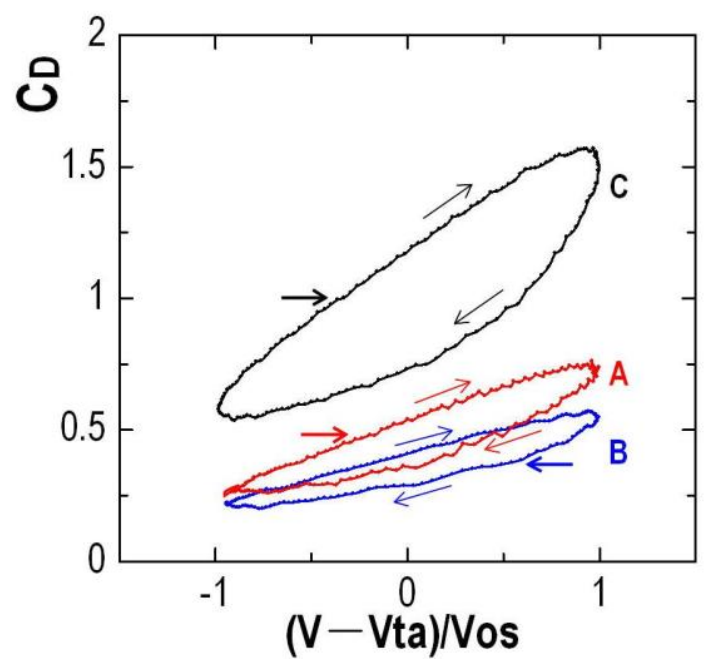

(a)

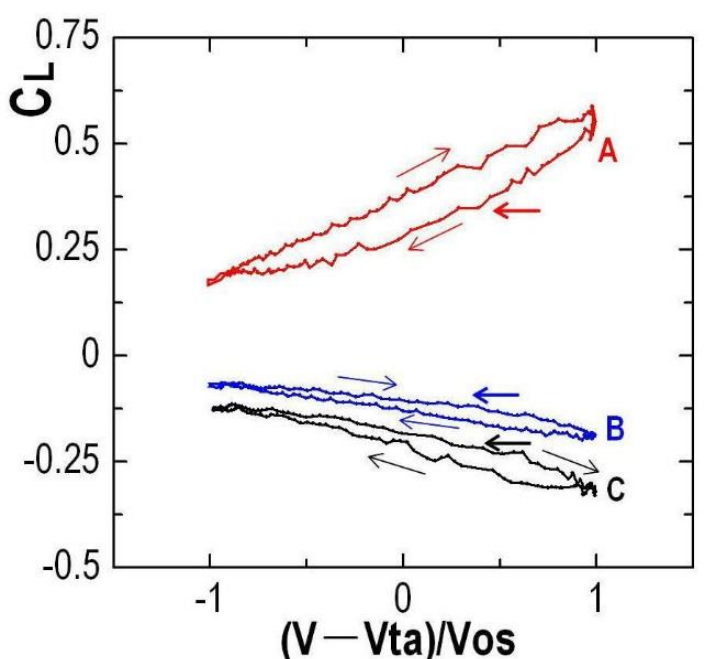

(b)

Figure 10. Lissajous diagram of (a) drag coefficient and; (b) lift coefficient (with A: Model A, B: Model B, C: Model C).

In the figures, the horizontal axis is the non-dimensionalised oscillating velocity of the wind, $\left(V-V_{t a}\right) / V_{o s}$. The horizontal arrows indicate the time-mean value.

The $C_{D}$ and $C_{L}$ change in a clockwise direction with time. As seen from Figure 10(a), the drag force increases approximately in proportion to the wind speed, when the headwind becomes strong. On the contrary, it decreases rapidly for the first half of the phase when the wind weakens. The difference in the drag forces between an increasing and a decreasing flow-speed is the largest in the case of model $\mathrm{C}$, with the horn-shaped front body. On the contrary, it is the smallest for model B.

In relation to the lift coefficient $C_{L}$, there is also a significant difference among the models, depending on the configuration of the rear body as in Figure 10(b). The influence of an increasing and decreasing wind on $C_{L}$ is the largest for model $\mathrm{A}$, while it is smaller for models B and C 


\section{CONCLUSION}

The characteristics of the fluid forces acting on-road vehicles modelled in three different shapes were investigated experimentally under pulsating wind conditions. The effects of wind oscillation and vehicle shape on the fluid forces were examined. The principal findings of this study are summarised as follows.

i. For the steady wind, the drag force of model $\mathrm{C}$ is roughly twice as large as that of Ahmed-type models A and B. On the other hand, the lift force acts downward in models $\mathrm{B}$ and $\mathrm{C}$ with symmetric upper and lower halves, and acts upward in model A, with a magnitude of around twice magnitudes of models B and C.

ii. The oscillation of the wind speed exerts a significant effect on the fluid forces, with the amplitude ratio of the forces being approximate twice the amplitude ratio of the wind velocity. Moreover, the drag and lift forces show a peculiar variation and complex behaviour, depending on the body shape of the vehicle model.

iii. The drag force increases in proportion to the wind speed as the headwind become stronger, while it rapidly decreases when the headwind begins to weaken. The larger the drag force on the vehicle model under a steady wind condition, the larger the variation of the drag force during a period. That is, in the rectangularshaped model C, the variation is the largest and in model B it is the smallest. Moreover, the drag force varies with a phase lead from the wind oscillation. In addition, its time-mean value is approximately $10 \%$ larger than the drag force under steady wind with the same value as the time-averaged velocity of the pulsating wind.

iv. The change in the lift force with time is different according to the model shape. Peculiarly, for models B and C with symmetric upper and lower halves, the lift force changes in the opposite phase of the wind oscillation. On the other hand, the difference in lift forces between an increasing and a decreasing wind-speed is the largest in the case of model A with a slant angle of $30^{\circ}$.

From those mentioned above the Ahmed-type model with a round front is, as expected, the preferred vehicle because of the low drag force. However, it will be necessary to furthermore investigate the influence of the rear slant angle on the aerodynamic characteristics.

There have been some papers [24, 25] concerning aerodynamic characteristics of road vehicles. However, the unsteady wind conditions have not been considered as the subject for research, although it is important in the real world. It is therefore desirable that researchers in the field of automotive engineering take an interest in this area of study.

\section{ACKNOWLEDGEMENT}

This work was supported by the MEXT-Supported Program for the Strategic Research Foundation at Private Universities (Grant No. S0901045), whose representative was Prof. H. Kyogoku, and in part by the Japan Climate Systems Corporation.

\section{REFERENCES}

[1] Kaneko M. Wind tunnel technologies for automobile development. Journal of the Visualization Society of Japan. 2016; 32(124): 9-13. 
[2] Tadakuma K, Haramoto Y, Murayama T, Maeda K. Development of full-scale wind tunnel to enhance aerodynamic and aero-acoustic performance. Journal of Society of Automotive Engineers of Japan. 2015; 69(7): 104-110.

[3] Kato D, Hashizume Y. Comparative method of vehicle aerodynamics between full-scale wind tunnel tests and on-road tests. Journal of Society of Automotive Engineers of Japan. 2015; 69(7): 111-116.

[4] Takagi M. Automotive wind tunnels. Journal of the Visualization Society of Japan. 2012; 32(124): 9-13.

[5] Baker CJ, Reynolds S. Wind-induced accidents of road vehicles. Accident Analysis \& Prevention. 1992; 24(6): 559-575.

[6] Chen SR, Cai CS. Accident assessment of vehicles on long-span bridges in windy environments. Journal of Wind Engineering and Industrial Aerodynamics. 2004; 92: 991-1024.

[7] Tsubokura M, Nakashima T. Aerodynamic effect on the high-speed straightahead stability and maneuverability of road vehicles. Journal of Society of Automotive Engineers of Japan. 2015; 69(7): 73-78.

[8] Liu X, Han Y, Cai CS, Levitan M, Nikitopoulos D. Wind tunnel tests for mean wind loads on road vehicles. Journal of Wind Engineering and Industrial Aerodynamics. 2016; 150: 15-21.

[9] Wordley S. Saunders J. On-road turbulence. SAE Paper No. 2008-01-0475; 2008.

[10] Fuller J, Best M, Garret N, Passmore M. The importance of unsteady aerodynamics to road vehicle dynamics. Journal of Wind Engineering and Industrial Aerodynamics. 2013; 117: 1-10.

[11] Kyogoku H. Report of study on development of automobile technologies in cooperation with university and the industry [MEXT-Supported Program (Grant No. S0901045)]-1 ${ }^{\text {st }}$ report: FY 2009 to 2010. Research Institute of Fundamental Technology for Next Generation, Kindai University, Higashi-hiroshima, Japan; 2011.

[12] Sumida M. KURING wind tunnel: aerodynamic characteristics tests of road vehicles in time-dependent winds. WSEAS Transactions on Fluid Mechanics. 2017; 12: 53-57.

[13] Greenblatt D. Unsteady low-speed wind tunnels. AIAA Journal. 2016; 54 (6): 1817-1830.

[14] Yagi T. Generation of unsteady flows in the wind tunnel. Wind Engineers, Japan Association for Wind Engineering. 2009; 34(1): 30-35.

[15] Ahmed SR, Ramm G. Some salient features of the time-averaged ground vehicle wake. SAE Paper No. 840300; 1984.

[16] Minguez M, Pasquetti R, Serre E. High-order large-eddy simulation of flow over the "Ahmed body" car model. Physics of Fluids 2008; 20: 095101.

[17] Meile W, Brenn G, Reppenhagen A, Lechner B, Fuchs A. Experiments and numerical simulations on the aerodynamics of the Ahmed body. CFD Letters. 2011; 3(1): 32-39.

[18] Lehmkuhl O, Borrell R, Rodriguez I, Perez-Segarra CD, Oliva A. Assessment of the symmetry-preserving regularization model on complex flows using unstructured grids. Computers \& Fluids. 2012; 60: 108-116.

[19] Wang XW, Zhou Y, Pin YF, Chan TL. Turbulent near wake of an Ahmed vehicle model. Experiments in Fluids. 2013; 54: 1490-1509.

[20] Tunay T, Sahin B, Akilli H. Experimental and numerical studies of the flow around the Ahmed body. Wind and Structures. 2013; 17(5): 515-535. 
[21] Aljure DE, Lehmkul O, Rodriguez I, Oliva A. Flow and turbulent structures around simplified car model. Computers \& Fluids. 2014; 96: 122-135.

[22] Banga S, Zunaid Md, Ansari NA, Sharma S, Dungriyal RS. CFD simulation of flow around external vehicle: Ahmed body. IOSR Journal of Mechanical and Civil Engineering. 2015; 12(4): 87-94.

[23] Uystepruyst D, Krajnovic S. LES of the flow around several cuboids in a row. International Journal of Heat and Fluid Flow. 2013; 44: 414-424.

[24] Ramasamy D, Mohanesan K, Kadirgama MMN, Rahman MM. Hybrid electric vehicle car body drag analysis using computational fluid dynamics. International Journal of Automotive and Mechanical Engineering. 2017; 14 (3): 4496-4507.

[25] Vedrtnam A, Sagar D. Variation of drag coefficient, wall static pressure and secondary flow over passenger car. International Journal of Automotive and Mechanical Engineering. 2018; 15 (1): 5002-5021. 